\title{
The Pathophysiology of Moyamoya Disease: An Update
}

\author{
Oh Young Bang, ${ }^{\text {a,d }}$ Miki Fujimura, ${ }^{b}$ Seung-Ki Kim ${ }^{c}$ \\ ${ }^{a}$ Department of Neurology, Samsung Medical Center, Sungkyunkwan University School of Medicine, Seoul, Korea \\ ${ }^{b}$ Department of Neurosurgery, Tohoku University Graduate School of Medicine, Sendai, Japan \\ 'Division of Pediatric Neurosurgery, Seoul National University Children's Hospital, Seoul National University College of Medicine, Seoul, Korea \\ ${ }^{\mathrm{d}}$ Translational and Stem Cell Research Laboratory on Stroke, Samsung Medical Center, Seoul, Korea
}

Moyamoya disease (MMD) is a unique cerebrovascular disease characterized by the progressive stenosis of large intracranial arteries and a hazy network of basal collaterals called moyamoya vessels. Because the etiology of MMD is unknown, its diagnosis is based on characteristic angiographic findings. Re-vascularization techniques (e.g., bypass surgery) are used to restore perfusion, and are the primary treatment for MMD. There is no specific treatment to prevent MMD progression. This review summarizes the recent advances in MMD pathophysiology, including the genetic and circulating factors related to disease development. Genetic and environmental factors may play important roles in the development of the vascular stenosis and aberrant angiogenesis in complex ways. These factors include the related changes in circulating endothelial/smooth muscle progenitor cells, cytokines related to vascular remodeling and angiogenesis, and endothelium, such as caveolin which is a plasma membrane protein. With a better understanding of MMD pathophysiology, nonsurgical approaches targeting MMD pathogenesis may be available to stop or slow the progression of this disease. The possible strategies include targeting growth factors, retinoic acid, caveolin-1, and stem cells.

Keywords Angiogenesis; Caveolin; Endothelial progenitor cells; Growth factors; Moyamoya disease

\author{
Correspondence: Oh Young Bang \\ Department of Neurology, Samsung \\ Medical Center, Sungkyunkwan \\ University, 81 Irwon-ro, Gangnam-gu, \\ Seoul 06351, Korea \\ Tel: +82-2-3410-3599 \\ Fax: +82-2-3410-1430 \\ E-mail: ohyoung.bang@samsung.com
}

Received: December 13, 2015

Revised: December 25, 2015

Accepted: December 30, 2015

This study was supported by the Korean Healthcare Technology R\&D Project, Ministry of Health \& Welfare (HI14C1531).

The authors have no financial conflicts of interest.

\section{Introduction}

Moyamoya disease (MMD) is a unique cerebrovascular disease characterized by the progressive stenosis of the distal internal carotid artery (ICA) and the resulting hazy network of basal collaterals called moyamoya vessels. The etiology of MMD is unknown. As a result, the criteria for the diagnosis of MMD is based on characteristic angiographic findings. However, the angiographic findings may not be sensitive or specific to MMD. The current diagnostic criteria require the presence of prominent basal collaterals for the diagnosis of MMD. However, a decision on the presence of basal collaterals can be subjective. In patients with MMD, the angiographic findings can differ according to the progressive stage and age of presentation, and the characteristic angiographic findings are not consistently observed in all courses of MMD..$^{1-3}$ In patients with an early stage of Suzuki's angiographic grading, the abnormal vascular network is not yet evident. ${ }^{4}$ Unlike in childhood-onset MMD, the basal collaterals are often not prominent in adult-onset MMD. ${ }^{3}$ In addition, the patients may present with unilateral MMD findings. In fact, the diagnostic criteria for definitive MMD was revised to include patients with both a bilateral and unilateral presentation of the terminal ICA stenosis with an abnormal vascular network at the base of 
the brain by the Research Committee of MMD of the Japanese Ministry of Health, Labour, and Welfare in 2015.

Owing to the currently limited understanding of MMD, revascularization techniques (e.g., bypass surgery) to restore perfusion are the primary treatment for MMD. There is no specific treatment to prevent $\mathrm{MMD}$ progression. The purpose of this review is to summarize the recent advances in MMD pathophysiology, including the genetic and circulating factors related to disease development.

\section{Pathological features of MMD}

The main pathological changes of the stenotic segment in MMD are the fibrocelluar thickening of the intima (e.g., the hyper-proliferation of the vessel wall components, active angiogenesis, and matrix accumulation), irregular undulation of the internal elastic laminae, medial thinness (e.g., an attenuation of media), and a decrease in the outer diameter. ${ }^{5-10}$

Recent neuroimaging techniques, such as the three-dimensional (3D) constructive interference in steady-state (CIISS) magnetic resonance imaging (MRI) and high-resolution MRI studies of patients with MMD, have demonstrated a constrictive remodeling (e.g., the narrowing of the arterial outer diameter) in affected segments and a concentric enhancement of the symptomatic segments. ${ }^{11-14}$ In our data set from a large cohort of adult-onset MMD, most patients (90.6\%) showed a long-segment concentric enhancement of the distal ICA and/ or middle cerebral artery on a high-resolution MRI, regardless of symptom presence or acuteness. The high-resolution MRI findings are consistent with the results of previous pathological reports that showed intimal hyperplasia and medial thinness. ${ }^{6,7}$

There is growing evidence that MMD is primarily a proliferative disease of the intima. The smooth muscle proliferation that is associated with an ACTA2 mutation has been postulated to be the key mechanism of the vascular occlusion in familial MMD. ${ }^{15}$ The histopathological findings in the distal ICA have shown a proliferation of the smooth muscle cells or endothelium ${ }^{8,10,16}$ and a stenosis or occlusion associated with the fibrocellular thickening of the intima. ${ }^{5}$ An enhancement of the stenotic segments may represent either a neo-vascularization or an intimal hyperplasia.

The moyamoya vessels are the dilated perforating arteries that have various histopathological changes, including fibrin deposits in the wall, fragmented elastic laminae, attenuated media, and the formation of microaneurysms. ${ }^{5}$ In addition to the moyamoya vessels, cortical microvascularization, which is characterized by a substantially increased microvascular density and diameter, is suggested as a specific finding in MMD. ${ }^{17}$
These basal and cortical vessels may represent compensatory mechanisms for the reduced cerebral blood flow or the aberrant active neo-vascularization before the vascular occlusion. An angiographic study of a large cohort of pediatric patients with MMD showed that the cortical neo-vascularization may occur before any significant hemodynamic impairment, suggesting that neo-vascularization is an active process, not a passive compensation for the vascular occlusion. ${ }^{18}$

The complicated pathologic features of the stenotic segments of MMD (e.g., a coexistence of proliferation and shrinkage) and the unknown nature of the neo-vascularization (e.g., an aberrant vs. compensatory process) suggest that MMD pathophysiology is a complex process.

\section{Genetics underlying MMD}

Approximately $10 \%$ of individuals with MMD exhibit a familial occurrence. Several genetic loci have been identified in familial MMD, including 3p24-26, ${ }^{19} 6 \mathrm{q} 25,{ }^{20} 8 \mathrm{q} 23,{ }^{21} 10 \mathrm{q} 23.31,{ }^{15}$ $12 \mathrm{p} 12,{ }^{21}$ and $17 \mathrm{q} 25 .{ }^{22}$ In addition, MMD is also associated with many genetically transmitted disorders, including neurofibromatosis, Down syndrome, sickle cell anemia, and collagen vascular disease. These findings suggest the importance of genetic factors.

\section{RNF213 as a susceptible gene for MMD}

More recently, the Ring finger 213 (RNF213) gene in the 17 q25-ter region was identified as the strongest susceptibility gene for MMD in East Asian people using a genome-wide linkage and exome analysis. ${ }^{23,24}$ The p.R4810K (c.14576G > A) variant of the RNF213 genetic variant was identified in $95 \%$ of patients with familial MMD, $80 \%$ with sporadic MMD, and $1.8 \%$ of control subjects in a Japanese population. ${ }^{23}$ The homozygous p.R4810K variant of RNF213 predicted an early onset and severe form of MMD in both Japanese ${ }^{25}$ and Kore$\mathrm{an}^{26}$ patients with MMD. The population that is susceptible to MMD, such as carriers of the RNF213 p.R4810K variant, is estimated to be 16.16 million people in East Asian countries. ${ }^{27}$ The number of patients with MMD, which was conservatively estimated at 1 per 300 carriers of the RNF213 p.R4810K variant, is considered to be 53,800 in East Asian populations. ${ }^{27,28}$

Further genetic studies for MMD are warranted, particularly in populations outside East Asia, because the single nucleotide polymorphism (SNP) of p.R4828K in RNF213 is not the susceptibility gene for MMD in Westerners or South Asian individuals. Novel variants in RNF213 in non-p.R4828K were recently found in Caucasian and Chinese cases with $\mathrm{MMD}^{24}$ and in the United States. ${ }^{29}$ For example, several variants of 
RNF213 in non-p.R4810K (i.e., rs148731719, rs397514563) were recently found in Caucasian and East and South Asian patients with $\mathrm{MMD} .^{24,29-31}$ In addition, the clinical manifestations and possibly angiographic findings may differ between Westerners and East Asians. ${ }^{32}$ The p.R4810K RNF213 variant was reportedly related to the ischemic type of MMD, while the non-p.R4810K RNF213 variants, particularly A4399T, were associated with the hemorrhagic-type of MMD. ${ }^{30}$

\section{Function of RNF213 on MMD pathophysiology}

The exact function of RNF213 is unknown. Recent in vivo experiments using genetically engineered RNF213 mice addressed the mechanism underlying the RNF213 SNPs in the development of MMD pathology. The target disruption of RNF213 did not induce MMD in the RNF213-defcient mice under normal conditions. ${ }^{33}$ Kanoke and colleagues alternatively generated RNF213-knock-in mice that expressed a missense mutation in the mouse RNF213, p.R4828K, on Exon 61, which corresponds to the human RNF213, p.R4859K, on Exon 60 in MMD patients; however, these mice did not develop MMD under normal conditions. ${ }^{34}$ These negative results could be consistent with the low penetrance rate of the RNF213 polymorphisms in patients with MMD, and may indicate the importance of environmental factors in addition to the genetic factors. ${ }^{35}$ They subjected the RNF213-deficient mice to an ischemic insult, and found that the post-ischemic angiogenesis was significantly enhanced in the mice lacking RNF213 after a chronic hindlimb ischemia. ${ }^{36}$ This suggests the potential role of a RNF213 abnormality in the development of abnormal vascular networks in chronic ischemia.

Hitomi et al. established a model of induced pluripotent stem cells derived from vascular endothelial cells (iPSECs), and showed that the angiogenic activity from patients with MMD and RNF213 carriers was lower than that of the control subjects. The overexpression of the RNF213 variant downregulated Securin and inhibited angiogenic activity. ${ }^{37}$ They also showed that RNF213 may be a mediator downstream of the IFN- $\beta$ signaling pathway in endothelial cells. Carriers of the RNF213 variant may be susceptible to cerebral hypoxia because of insufficient angiogenesis if inflammation and hypoxia occur simultaneously. ${ }^{38}$ Ohkubo and colleagues also showed that pro-inflammatory cytokines activated RNF213 transcription, and RNF213 functions as a common downstream effector of the PI3 kinase-AKT pathway in endothelial angiogenesis. ${ }^{39}$ These data suggest that although MMD is not an inflammatory disease, inflammation may play an important role in MMD development. RNF213 plays a unique role in endothelial cells regarding the proper gene expression in re- sponse to inflammatory signals from the environment. However, further studies are needed to elucidate the differential pathological processes between the endothelium (e.g., lowered angiogenesis) and smooth muscle cells (e.g., abnormal proliferation that causes moyamoya vessel formation and stenosis of the major intracranial arteries).

In addition to the preclinical data, clinical data has also shown that exposure to environmental factors, such as an autoimmune response and infection/inflammation, in MMDsusceptible subjects may be associated with the angiographic features of MMD. ${ }^{35}$ For example, autoimmune thyroid disease has been reported in different MMD populations (i.e., pediatric and adult-onset MMD, East Asians, and Westerners) and may be involved in MMD development. ${ }^{40-42}$ In addition, the RNF213 genetic variant may be associated with vascular risk factors, such as hypertension, ${ }^{43}$ and also could lead to vascular fragility, which may make vessels more vulnerable to hemodynamic stress and secondary insults. ${ }^{35}$

\section{Polymorphisms of microRNAs}

MicroRNAs (miRs), which are small non-coding RNAs ( $\sim 23$ nucleopeptides), negatively regulate the expression of many proteins by altering their gene expression through posttranscriptional repression or mRNA degradation. ${ }^{44}$ miRs may play an essential role in the regulation of proliferation, differentiation, survival, and aging of various tissues and cells, including stem cells. There is increasing evidence that miRs that are altered after focal ischemia have a functional significance in the recovery after stroke as well as ischemic pathophysiology. Preclinical studies of ischemic stroke have demonstrated that miRs protect against focal ischemia and reperfusion injury by inhibiting oxidative stress. ${ }^{45}$ They are also involved in inflammation, neurogenesis, and angiogenesis. ${ }^{46-48}$

A genome-wide miR array analysis of the serum from patients with MMD showed elevated serum levels of miRs associated with RNF213 and BRCC3 (i.e., BRCA1/BRCA2-containing complex, an important angiogenesis-related protein), both of which are involved in MMD pathogenesis. ${ }^{49}$ In addition, a SNP of miR-196a was associated with MMD. ${ }^{50}$ ANXA1, which is expressed in endothelial and smooth muscle cells, ${ }^{51}$ is a gene target of miR196a and mediates the apoptosis and inhibition of cell proliferation. ${ }^{52}$

\section{Biomarkers underlying vascular stenosis and aberrant angiogenesis}

In addition to genetic biomarkers, there are circulating factors that may be involved in MMD pathogenesis, including cir- 
culating endothelial progenitor cells (EPCs), cytokines, and caveolin.

\section{Circulating vascular progenitor cells}

In patients with acute myocardial infarcts or ischemic stroke, increasing evidence points to a role for circulating EPCs that originate from the bone marrow and help maintain the vasculature and blood flow in an infarcted area. ${ }^{53}$ EPCs potentially contribute to the neo-vascularization at the ischemic brain injury site in patients with MMD. ${ }^{54}$ Rafat et al. reported the presence of increased levels of circulating EPCs in patients with MMD..${ }^{55}$ In contrast, Kim et al. demonstrated decreased EPC levels and defective angiogenic function in EPCs in pediatric patients with $\mathrm{MMD}$, indicating there is abnormal angiogenesis during MMD pathogenesis. ${ }^{56}$ Similarly, impaired EPC function was observed in adult patients with MMD. ${ }^{57}$ Recently, Lee et al. reported a downregulation of retinaldehyde dehydrogenase 2 (RALDH2) using the gene expression profiles of EPC in pediatric patients with MMD. The epigenetic suppression of RALDH2 expression contributed to the defective function of MMD endothelial colony-forming cells; this could be rescued by supplying retinoic acid in vitro and in vivo. ${ }^{58}$ Aberrant angiogenesis was an active angiogenetic process that may cause both stenosis through the proliferation of endothelial and/or smooth muscle cells and abnormal collateral formation. ${ }^{8}$

In addition to endothelial cells, the smooth muscle cells are also involved in this disease process. The MMD pathology is characterized by smooth muscle cell hyperplasia in the intima. Mutations in the smooth muscle cells, such as smooth muscle alpha-actin, which is encoded by ACTA2, may be involved in the increased proliferation of the smooth muscle cells, contributing to occlusive diseases. ${ }^{15}$ Recently, Kang and colleagues cultured and isolated smooth muscle progenitor cells (SPCs) from the peripheral blood of patients with $\mathrm{MMD}$, and showed that the SPCs in the MMD group tended to make more irregularly arranged and thickened tubules, as well as express differential genes compared to that of the healthy controls. ${ }^{59}$ These findings suggest a defect in the cell maturation process that might have occurred in the SPCs from the patients with MMD.

\section{Cytokine and their polymorphisms}

Various cytokines and their polymorphisms are associated with MMD, including (a) growth factors, such as vascular endothelial growth factor (VEGF), fibroblast growth factor, platelet-derived growth factor (PDGF), and hepatocyte growth factor, (b) cytokines related to vascular remodeling and angiogenesis, such as matrix metalloproteinases (MMPs) and their inhibitors, hypoxia-inducible factor- $1 \alpha$, and cellular retinoic binding protein-1 (CRABP-1), and (c) cytokines related to inflammation. ${ }^{55,60-64}$ The investigations regarding the role of these factors have been inconclusive.

A genetic study of familial MMD investigated the balance between MMPs and their inhibitors, and found that the presence of a certain MMP inhibitor genotype may be a predisposing genetic factor for familial MMD. ${ }^{65}$ The levels of several trophic factors, such as VEGF, basic fibroblast growth factor, and PDGF-BB, were increased, but the VEGF receptor levels were decreased in MMD compared to that of controls. ${ }^{60,66,67} \mathrm{In}$ addition, certain VEGF polymorphisms were associated with pediatric MMD and poor collateral vessel formation. ${ }^{68}$ However, these findings were not observed in other studies. ${ }^{69}$ Using a multifactor dimensionality reduction method, a recent study evaluated the interactions of different loci for MMD, but failed to show any influence of $\beta$-type PDGF receptor and MMPs on MMD. ${ }^{69}$ Young et al. suggested that the induction of pro-inflammation cascades and VEGF expression is secondary to the infarct rather than part of the primary MMD pathology. ${ }^{70}$

Changes in the levels of these factors may be simply associated with the disease rather than causative, as many of these factors are also increased in patients with stroke. However, Kim and colleagues identified a polypeptide spot, CRABP-1, in cerebrospinal fluid from pediatric patients with MMD using a proteomics analysis. ${ }^{63} \mathrm{~A}$ higher CRABP-1 level in the CSF was associated with a typical bilateral involvement and a decrease in the basal collaterals post-operatively in adult MMD. ${ }^{71}$ It has been proposed that the retinoids attenuate growth factor-stimulated smooth muscle cell migration and proliferation, and CRABP-1 can negatively regulate retinoic acid activity. ${ }^{63}$ These findings suggest an important role for retinoid signaling in MMD pathogenesis by controlling the growth factor expression. ${ }^{63}$ Further studies are needed to determine whether retinoids are efficacious for MMD treatment. Although pathological analyses have revealed that the affected vessels do not show any inflammatory changes that lead to occlusion, ${ }^{16}$ the role of inflammation in the fibrocellular thickening of the intima and the disease pathogenesis are also being investigated. The plasma levels of MMPs, monocyte chemoattractant protein-1, and inflammatory cytokines (interleukin-1b) were higher in patients with MMD compared to those in controls. ${ }^{60} \mathrm{~A}$ previous study, ${ }^{72}$ as well as our unpublished data, showed that the levels of E-selectin, which is involved in endothelial progenitor cell recruitment and angiogenesis) were increased in both patients with $\mathrm{MMD}$ and those with atherosclerotic stroke. 


\section{Endothelial function and nitric oxide metabolites}

Endothelial dysfunction is responsible for the dysregulation of vascular tone, cellular adhesion, thrombus formation, smooth muscle cell proliferation, and vessel wall inflammation. MMD may be a condition that is more vulnerable to environmental influences that cause endothelial dysfunction. A previous study of a small cohort showed that the nitric oxide (NO) metabolites (i.e., nitrate and nitrite) were increased in cerebrospinal flow samples of 18 patients with MMD compared to that of controls, which probably reflects the development of abnormal collateral circulation. ${ }^{73}$ However, further studies are needed to confirm this result. In our unpublished data, the serum levels of NO metabolites and asymmetric dimethylarginine (ADMA; an endogenous competitive antagonist of NO synthase, a marker of endothelial dysfunction) in patients with MMD did not differ from those of control subjects. In addition, no differences were observed between control and MMD in endothelial NO synthase polymorphisms. ${ }^{74}$

\section{Caveolin and dysfunction in endothelial vesicular trafficking and signal transduction}

Caveolae are 50-100 nm cell surface plasma membrane invaginations that are abundant in endothelial cells, and play a major role in the regulation of endothelial vesicular trafficking and signal transduction. ${ }^{75}$ Caveolin-1, a scaffolding protein of the caveolae plasma membrane, is involved in the pathogenesis of cancers and vascular diseases. ${ }^{75}$ Caveolin-1 overexpression enhanced caveolae generation and accelerated the capillary tube formation by nearly three-fold, while caveolin-1 downregulation reduced the in vitro and in vivo capillary formation, and was associated with pathological angiogenesis. ${ }^{75-77}$ Both endothelial NO synthase and VEGFR2 co-localized in the caveolae, while caveolin-1 expression was critical for VEGF-induced angiogenesis in an ischemic hind limb model ${ }^{78}$ and endothelial NO-related tumor angiogenesis. ${ }^{79}$ One study that used an ischemic hind limb model demonstrated that caveolin-1 was also involved in endothelial progenitor cell recruitment from the bone marrow. ${ }^{80}$

Our recent study showed that caveolin-1 is a key mediator for MMD (unpublished data). In this study, the caveolin-1 serum levels decreased in adult patients with MMD and were markedly decreased in those with the RNF213 variant. Liu and colleagues showed the differential roles of caveolin-1 during the differential phases of angiogenesis, such as caveolin-1 negatively regulating an earlier phase of angiogenesis (i.e., endothelial cell proliferation), but positively regulating a later phase of angiogenesis (i.e., tube formation) ${ }^{76}$ Collectively, the decreased caveolin-1 levels and resulting increased prolifera-

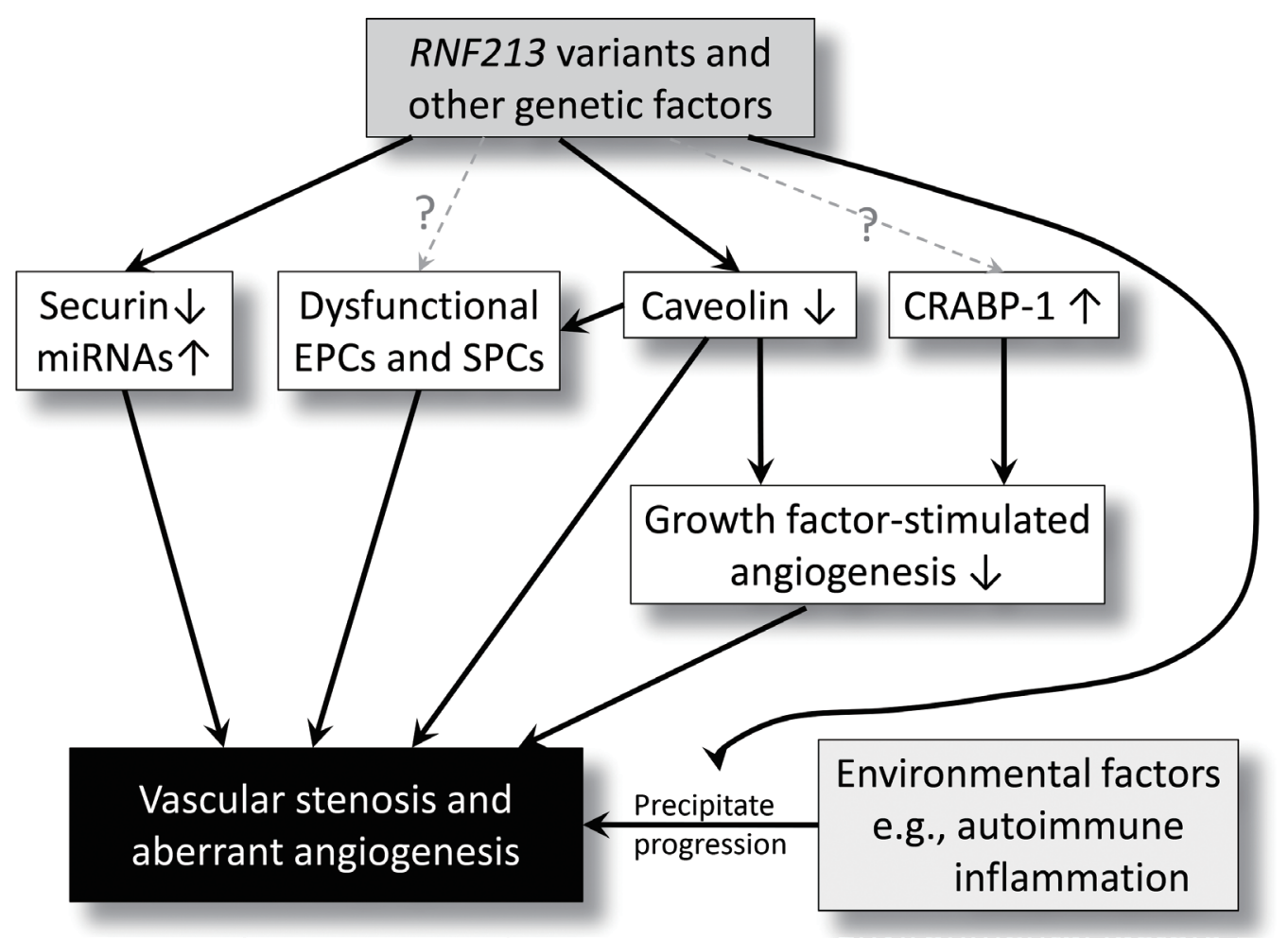

Figure 1. Potential mechanisms of moyamoya disease. The association between genetic, circulating, and environmental factors. RNF213, Ring finger 213; EPCs, endothelial progenitor cells; SPCS, smooth muscle progenitor cells; miRNAs, microRNAs; CRABP-1, cellular retinoic acid-binding protein-I. 
tion and decreased stabilization/tube formation in patients with MMD suggests that the nature of neo-vascularization is an aberrant rather than a compensatory process. The elucidation of mechanisms of the caveolin-1-related pathological angiogenesis may pave the way for various therapeutic strategies. ${ }^{80,81}$ Caveolin expression could be modulated by genetic regulation targeting caveolin-1, such as antisense/siRNA or microRNA, anti-caveolin-1 antibodies, viral vectors or polymers that target the caveolae. Further studies are needed because most previous studies were performed in ischemic hind limb or cancer models. One study investigated the effects of cerebral ischemia in caveolin-1 knockout mice and demonstrated impaired angiogenesis and increased apoptotic cell death. ${ }^{82}$ In addition to angiogenesis, a series of signaling pathways couple caveolae with ischemia (e.g., neuroinflammation, blood-brain barrier permeability, and pre-conditioning), and caveolin was recently suggested as a novel therapeutic target for ischemic stroke. ${ }^{83}$

\section{Conclusion and perspectives}

Although the pathogenic mechanisms of MMD are still unknown, there is growing evidence that it is primarily a proliferative disease, such that endothelial and smooth muscle proliferation results in the development of an occlusion, and enhanced, but aberrant, angiogenesis (i.e., moyamoya vessels). Genetic factors and related changes in circulating factors, as well as environmental factors, may play important roles in complex ways (Figure 1).

Further studies are needed because there is no relevant MMD animal model using these factors. In addition, these genetic and related changes in circulating factors would confer pathophysiological effects on the systemic vessels as well as distal ICA/proximal middle cerebral artery. They cannot explain the site specificity with sparing systemic vessels. Moreover, none of the previous reports have studied the levels of circulating biomarkers in relation to the disease stage. The profile of circulating biomarkers may differ according to the disease stage.

At present, surgical re-vascularization is the mainstay MMD treatment. However, surgical treatments pose a possible risk for peri-operative ischemic complications and/or cerebral hyperperfusion syndrome. ${ }^{84}$ With a better understanding of MMD pathophysiology, non-surgical approaches targeting MMD pathogenesis may be available to stop or slow the progression of this disease. Non-surgical approaches may include the application of (a) certain trophic factors or chemicals that increase angiogenesis, ${ }^{85}$ (b) anti-cancer drugs to decrease the smooth muscle cell proliferation, ${ }^{15}$ (c) retinoid to attenuate growth factor-stimulated smooth muscle cell migration and proliferation, ${ }^{58,63}(\mathrm{~d})$ several strategies to increase caveolin-1 levels, ${ }^{81}$ and (e) stem cell therapy to replace or restore function of impaired EPCs or SPCs. Further efforts will benefit from collaborative works between the clinical hospital bed and the laboratory bench.

\section{References}

1. Scott RM, Smith ER. Moyamoya disease and moyamoya syndrome. N Engl J Med 2009;360:1226-1237.

2. Fukui M. Guidelines for the diagnosis and treatment of spontaneous occlusion of the circle of willis ('moyamoya' disease). Research committee on spontaneous occlusion of the circle of willis (moyamoya disease) of the ministry of health and welfare, japan. Clin Neurol Neurosurg 1997;99 Suppl 2:S238-240.

3. Bang OY, Ryoo S, Kim SJ, Yoon CH, Cha J, Yeon JY, et al. Adult moyamoya disease: a burden of intracranial stenosis in east asians? PLoS One 2015; 10:e0130663.

4. Suzuki J, Takaku A. Cerebrovascular "moyamoya” disease. Disease showing abnormal net-like vessels in base of brain. Arch Neurol 1969;20:288-299.

5. Kuroda S, Houkin K. Moyamoya disease: current concepts and future perspectives. Lancet Neurol 2008;7:1056-1066.

6. Takagi Y, Kikuta K, Nozaki K, Hashimoto N. Histological features of middle cerebral arteries from patients treated for moyamoya disease. Neurol Med Chir (Tokyo) 2007;47:1-4.

7. Oka K, Yamashita M, Sadoshima S, Tanaka K. Cerebral haemorrhage in moyamoya disease at autopsy. Virchows Archiv. A, Pathological Anatomy and Histology 1981;392:247-261.

8. Chmelova J, Kolar Z, Prochazka V, Curik R, Dvorackova J, Sirucek $\mathrm{P}$, et al. Moyamoya disease is associated with endothelial activity detected by anti-nestin antibody. Biomedical papers of the Medical Faculty of the University Palacky, Olomouc, Czechoslovakia 2010;154:159-162.

9. Lin R, Xie Z, Zhang J, Xu H, Su H, Tan X, et al. Clinical and immunopathological features of moyamoya disease. PLoS One 2012; 7:e36386.

10. Takagi Y, Kikuta K, Nozaki K, Fujimoto M, Hayashi J, Imamura $\mathrm{H}$, et al. Expression of hypoxia-inducing factor-1 alpha and endoglin in intimal hyperplasia of the middle cerebral artery of patients with moyamoya disease. Neurosurgery 2007;60:338345; discussion 345.

11. Kaku Y, Morioka M, Ohmori Y, Kawano T, Kai Y, Fukuoka H, et al. Outer-diameter narrowing of the internal carotid and middle cerebral arteries in moyamoya disease detected on $3 \mathrm{~d}$ constructive interference in steady-state $\mathrm{mr}$ image: is arterial 
constrictive remodeling a major pathogenesis? Acta Neurochir (Wien) 2012;154:2151-2157.

12. Kim YJ, Lee DH, Kwon JY, Kang DW, Suh DC, Kim JS, et al. High resolution mri difference between moyamoya disease and intracranial atherosclerosis. Eur J Neurol 2013;20:1311-1318.

13. Ryoo S, Cha J, Kim SJ, Choi JW, Ki CS, Kim KH, et al. Highresolution magnetic resonance wall imaging findings of moyamoya disease. Stroke 2014;45:2457-2460.

14. Yuan M, Liu ZQ, Wang ZQ, Li B, Xu LJ, Xiao XL. High-resolution $\mathrm{mr}$ imaging of the arterial wall in moyamoya disease. $\mathrm{Neu}$ rosci Lett 2015;584:77-82.

15. Guo DC, Papke CL, Tran-Fadulu V, Regalado ES, Avidan N, Johnson RJ, et al. Mutations in smooth muscle alpha-actin (acta2) cause coronary artery disease, stroke, and moyamoya disease, along with thoracic aortic disease. Am J Hum Genet 2009;84:617-627.

16. Fukui M, Kono S, Sueishi K, Ikezaki K. Moyamoya disease. Neuropathology 2000;20 Suppl:S61-64.

17. Czabanka M, Pena-Tapia P, Schubert GA, Woitzik J, Vajkoczy P, Schmiedek P. Characterization of cortical microvascularization in adult moyamoya disease. Stroke 2008;39:1703-1709.

18. Kim SJ, Son TO, Kim KH, Jeon P, Hyun SH, Lee KH, et al. Neovascularization precedes occlusion in moyamoya disease: angiographic findings in 172 pediatric patients. Eur Neurol 2014; 72:299-305.

19. Ikeda H, Sasaki T, Yoshimoto T, Fukui M, Arinami T. Mapping of a familial moyamoya disease gene to chromosome 3p24.2p26. Am J Hum Genet 1999;64:533-537.

20. Inoue TK, Ikezaki K, Sasazuki T, Matsushima T, Fukui M. Linkage analysis of moyamoya disease on chromosome 6. J Child Neurol 2000;15:179-182.

21. Sakurai K, Horiuchi Y, Ikeda H, Ikezaki K, Yoshimoto T, Fukui $\mathrm{M}$, et al. A novel susceptibility locus for moyamoya disease on chromosome 8q23. J Hum Genet 2004;49:278-281.

22. Yamauchi T, Tada M, Houkin K, Tanaka T, Nakamura Y, Kuroda $S$, et al. Linkage of familial moyamoya disease (spontaneous occlusion of the circle of willis) to chromosome 17q25. Stroke 2000;31:930-935.

23. Kamada F, Aoki Y, Narisawa A, Abe Y, Komatsuzaki S, Kikuchi A, et al. A genome-wide association study identifies $\operatorname{rnf} 213$ as the first moyamoya disease gene. J Hum Genet 2011;56:34-40.

24. Liu W, Morito D, Takashima S, Mineharu Y, Kobayashi H, Hitomi $\mathrm{T}$, et al. Identification of $\mathrm{rnf} 213$ as a susceptibility gene for moyamoya disease and its possible role in vascular development. PLoS One 2011;6:e22542.

25. Miyatake S, Miyake N, Touho H, Nishimura-Tadaki A, Kondo Y, Okada I, et al. Homozygous c.14576g >a variant of $\operatorname{rnf} 213$ predicts early-onset and severe form of moyamoya disease.
Neurology 2012;78:803-810.

26. Kim EH, Yum MS, Ra YS, Park JB, Ahn JS, Kim GH, et al. Importance of $\mathrm{rnf} 213$ polymorphism on clinical features and longterm outcome in moyamoya disease. J Neurosurg 2015:1-7.

27. Liu W, Hitomi T, Kobayashi H, Harada KH, Koizumi A. Distribution of moyamoya disease susceptibility polymorphism p.R4810k in rnf213 in east and southeast asian populations. Neurol Med Chir (Tokyo) 2012;52:299-303.

28. Liu W, Hashikata H, Inoue K, Matsuura N, Mineharu Y, Kobayashi $\mathrm{H}$, et al. A rare asian founder polymorphism of raptor may explain the high prevalence of moyamoya disease among east asians and its low prevalence among caucasians. Environmental Health and Preventive Medicine 2010;15:94-104.

29. Cecchi AC, Guo D, Ren Z, Flynn K, Santos-Cortez RL, Leal $\mathrm{SM}$, et al. Rnf213 rare variants in an ethnically diverse population with moyamoya disease. Stroke 2014;45:3200-3207.

30. Wu Z, Jiang H, Zhang L, Xu X, Zhang X, Kang Z, et al. Molecular analysis of rnf213 gene for moyamoya disease in the chinese han population. PLoS One 2012;7:e48179.

31. Ma J, Liu Y, Ma L, Huang S, Li H, You C. Rnf213 polymorphism and moyamoya disease: a systematic review and metaanalysis. Neurology India 2013;61:35-39.

32. Kleinloog R, Regli L, Rinkel GJ, Klijn CJ. Regional differences in incidence and patient characteristics of moyamoya disease: a systematic review. J Neurol Neurosurg Psychiatry 2012;83: 531-536.

33. Sonobe S, Fujimura M, Niizuma K, Nishijima Y, Ito A, Shimizu $\mathrm{H}$, et al. Temporal profile of the vascular anatomy evaluated by 9.4-t magnetic resonance angiography and histopathological analysis in mice lacking rnf213: a susceptibility gene for moyamoya disease. Brain Res 2014;1552:64-71.

34. Kanoke A, Fujimura M, Niizuma K, Ito A, Sakata H, Sato-Maeda $\mathrm{M}$, et al. Temporal profile of the vascular anatomy evaluated by 9.4-tesla magnetic resonance angiography and histological analysis in mice with the r4859k mutation of rnf 213 , the susceptibility gene for moyamoya disease. Brain Research 2015; 1624:497-505.

35. Fujimura M, Sonobe S, Nishijima Y, Niizuma K, Sakata H, Kure S, et al. Genetics and biomarkers of moyamoya disease: Significance of $\mathrm{rnf} 213$ as a susceptibility gene. Journal of Stroke 2014;16:65-72.

36. Ito A, Fujimura M, Niizuma K, Kanoke A, Sakata H, MoritaFujimura $Y$, et al. Enhanced post-ischemic angiogenesis in mice lacking rnf213; a susceptibility gene for moyamoya disease. Brain Res 2015;1594:310-320.

37. Hitomi T, Habu T, Kobayashi H, Okuda H, Harada KH, Osafune $\mathrm{K}$, et al. Downregulation of securin by the variant $\operatorname{rnf} 213$ r4810k (rs112735431, g>a) reduces angiogenic activity of in- 
duced pluripotent stem cell-derived vascular endothelial cells from moyamoya patients. Biochem Biophys Res Commun 2013; 438:13-19.

38. Kobayashi H, Matsuda Y, Hitomi T, Okuda H, Shioi H, Matsuda $\mathrm{T}$, et al. Biochemical and functional characterization of $\mathrm{rnf} 213$ (mysterin) r4810k, a susceptibility mutation of moyamoya disease, in angiogenesis in vitro and in vivo. J Am Heart Assoc 2015; 4:e002146.

39. Ohkubo K, Sakai Y, Inoue H, Akamine S, Ishizaki Y, Matsushita $\mathrm{Y}$, et al. Moyamoya disease susceptibility gene rnf213 links inflammatory and angiogenic signals in endothelial cells. Sci Rep 2015;5:13191.

40. Kim SJ, Heo KG, Shin HY, Bang OY, Kim GM, Chung CS, et al. Association of thyroid autoantibodies with moyamoya-type cerebrovascular disease: A prospective study. Stroke 2010;41: 173-176.

41. Bower RS, Mallory GW, Nwojo M, Kudva YC, Flemming KD, Meyer FB. Moyamoya disease in a primarily white, midwestern us population: increased prevalence of autoimmune disease. Stroke 2013;44:1997-1999.

42. Li H, Zhang ZS, Dong ZN, Ma MJ, Yang WZ, Han C, et al. Increased thyroid function and elevated thyroid autoantibodies in pediatric patients with moyamoya disease: a case-control study. Stroke 2011;42:1138-1139.

43. Koizumi A, Kobayashi H, Liu W, Fujii Y, Senevirathna ST, Nanayakkara S, et al. P.R4810k, a polymorphism of $\mathrm{rnf} 213$, the susceptibility gene for moyamoya disease, is associated with blood pressure. Environ Health Prev Med 2013;18:121-129.

44. Bartel DP. Micrornas: genomics, biogenesis, mechanism, and function. Cell 2004;116:281-297.

45. Liu P, Zhao H, Wang R, Wang P, Tao Z, Gao L, et al. Microrna-424 protects against focal cerebral ischemia and reperfusion injury in mice by suppressing oxidative stress. Stroke 2015;46: 513-519.

46. De Rosa S, Curcio A, Indolfi C. Emerging role of micrornas in cardiovascular diseases. Circulation Journal: Official Journal of the Japanese Circulation Society 2014;78:567-575.

47. Koutsis G, Siasos G, Spengos K. The emerging role of microrna in stroke. Current Topics in Medicinal Chemistry 2013;13: 1573-1588.

48. Zeng L, He X, Wang Y, Tang Y, Zheng C, Cai H, et al. Microrna-210 overexpression induces angiogenesis and neurogenesis in the normal adult mouse brain. Gene Therapy 2014;21:37-43.

49. Dai D, Lu Q, Huang Q, Yang P, Hong B, Xu Y, et al. Serum mirna signature in moyamoya disease. PLoS One 2014;9:e102382.

50. Park YS, Jeon YJ, Lee BE, Kim TG, Choi JU, Kim DS, et al. Association of the mir-146ac $>$ g, mir-196a2c>t, and mir-499a>g polymorphisms with moyamoya disease in the korean popula- tion. Neurosci Lett 2012;521:71-75.

51. Eberhard DA, Brown MD, VandenBerg SR. Alterations of annexin expression in pathological neuronal and glial reactions. Immunohistochemical localization of annexins i, ii (p36 and p11 subunits), iv, and vi in the human hippocampus. Am J Pathol 1994;145:640-649.

52. Herbert SP, Odell AF, Ponnambalam S, Walker JH. The confluence-dependent interaction of cytosolic phospholipase a2alpha with annexin al regulates endothelial cell prostaglandin e2 generation. J Biol Chem 2007;282:34468-34478.

53. Kumar AH, Caplice NM. Clinical potential of adult vascular progenitor cells. Arterioscler Thromb Vasc Biol 2010;30:10801087.

54. Yoshihara T, Taguchi A, Matsuyama T, Shimizu Y, KikuchiTaura A, Soma $\mathrm{T}$, et al. Increase in circulating cd34-positive cells in patients with angiographic evidence of moyamoya-like vessels. J Cereb Blood Flow Metab 2008;28:1086-1089.

55. Rafat N, Beck G, Pena-Tapia PG, Schmiedek P, Vajkoczy P. Increased levels of circulating endothelial progenitor cells in patients with moyamoya disease. Stroke 2009;40:432-438.

56. Kim JH, Jung JH, Phi JH, Kang HS, Kim JE, Chae JH, et al. Decreased level and defective function of circulating endothelial progenitor cells in children with moyamoya disease. $J \mathrm{Neu}$ rosci Res 2010;88:510-518.

57. Jung KH, Chu K, Lee ST, Park HK, Kim DH, Kim JH, et al. Circulating endothelial progenitor cells as a pathogenetic marker of moyamoya disease. J Cereb Blood Flow Metab 2008;28: 1795-1803.

58. Lee JY, Moon YJ, Lee HO, Park AK, Choi SA, Wang KC, et al. Deregulation of retinaldehyde dehydrogenase 2 leads to defective angiogenic function of endothelial colony-forming cells in pediatric moyamoya disease. Arterioscler Thromb Vasc Biol 2015; 35:1670-1677.

59. Kang HS, Moon YJ, Kim YY, Park WY, Park AK, Wang KC, et al. Smooth-muscle progenitor cells isolated from patients with moyamoya disease: Novel experimental cell model. J Neurosurg 2014;120:415-425.

60. Kang HS, Kim JH, Phi JH, Kim YY, Kim JE, Wang KC, et al. Plasma matrix metalloproteinases, cytokines and angiogenic factors in moyamoya disease. J Neurol Neurosurg Psychiatry 2010;81:673-678.

61. Nanba R, Kuroda S, Ishikawa T, Houkin K, Iwasaki Y. Increased expression of hepatocyte growth factor in cerebrospinal fluid and intracranial artery in moyamoya disease. Stroke 2004;35: 2837-2842.

62. Park YS, Jeon YJ, Kim HS, Chae KY, Oh SH, Han IB, et al. The role of vegf and $\mathrm{kdr}$ polymorphisms in moyamoya disease and collateral revascularization. PLoS One 2012;7:e47158. 
63. Kim SK, Yoo JI, Cho BK, Hong SJ, Kim YK, Moon JA, et al. Elevation of crabp-i in the cerebrospinal fluid of patients with moyamoya disease. Stroke 2003;34:2835-2841.

64. Fujimura M, Watanabe M, Narisawa A, Shimizu H, Tominaga T. Increased expression of serum matrix metalloproteinase- 9 in patients with moyamoya disease. Surg Neurol 2009;72:476480; discussion 480.

65. Kang HS, Kim SK, Cho BK, Kim YY, Hwang YS, Wang KC. Single nucleotide polymorphisms of tissue inhibitor of metalloproteinase genes in familial moyamoya disease. Neurosurgery 2006;58:1074-1080; discussion 1074-1080.

66. He J, Wang R, Zhang D, Zhang Y, Zhang Q, Zhao J. Expression of circulating vascular endothelial growth factor-antagonizing cytokines and vascular stabilizing factors prior to and following bypass surgery in patients with moyamoya disease. Exp Ther Med 2014;8:302-308.

67. Hoshimaru M, Takahashi JA, Kikuchi H, Nagata I, Hatanaka M. Possible roles of basic fibroblast growth factor in the pathogenesis of moyamoya disease: an immunohistochemical study. JNeurosurg 1991;75:267-270.

68. Park YS, Jeon YJ, Kim HS, Chae KY, Oh SH, Han IB, et al. The role of vegf and $\mathrm{kdr}$ polymorphisms in moyamoya disease and collateral revascularization. PLoS One 2012; 7:e47158.

69. Wang X, Zhang Z, Liu W, Xiong Y, Sun W, Huang X, et al. Impacts and interactions of pdgfrb, mmp-3, timp-2, and rnf213 polymorphisms on the risk of moyamoya disease in han chinese human subjects. Gene 2013;526:437-442.

70. Young AM, Karri SK, Ogilvy CS, Zhao N. Is there a role for treating inflammation in moyamoya disease?: a review of histopathology, genetics, and signaling cascades. Frontiers in $\mathrm{Neu}$ rology 2013;4:105.

71. Jeon JS, Ahn JH, Moon YJ, Cho WS, Son YJ, Kim SK, et al. Expression of cellular retinoic acid-binding protein-i (crabp-i) in the cerebrospinal fluid of adult onset moyamoya disease and its association with clinical presentation and postoperative haemodynamic change. J Neurol Neurosurg Psychiatry 2014;85:726-731.

72. Soriano SG, Cowan DB, Proctor MR, Scott RM. Levels of soluble adhesion molecules are elevated in the cerebrospinal fluid of children with moyamoya syndrome. Neurosurgery 2002;50: 544-549.

73. Noda A, Suzuki Y, Takayasu M, Watanabe K, Takagi T, Hara $\mathrm{M}$, et al. Elevation of nitric oxide metabolites in the cerebrospinal fluid of patients with moyamoya disease. Acta Neurochirurgica 2000;142:1275-1279; discussion 1279-1280.

74. Park YS, Min KT, Kim TG, Lee YH, Cheong HJ, Yeom IS, et al.
Age-specific enos polymorphisms in moyamoya disease. Childs Nerv Syst 2011;27:1919-1926.

75. Frank PG, Woodman SE, Park DS, Lisanti MP. Caveolin, caveolae, and endothelial cell function. Arterioscler Thromb Vasc Biol 2003;23:1161-1168.

76. Liu J, Wang XB, Park DS, Lisanti MP. Caveolin-1 expression enhances endothelial capillary tubule formation. The Journal of Biological Chemistry 2002;277:10661-10668.

77. Chang SH, Feng D, Nagy JA, Sciuto TE, Dvorak AM, Dvorak HF. Vascular permeability and pathological angiogenesis in caveolin-1-null mice. The American Journal of Pathology 2009; 175:1768-1776.

78. Sonveaux P, Martinive P, DeWever J, Batova Z, Daneau G, Pelat $\mathrm{M}$, et al. Caveolin-1 expression is critical for vascular endothelial growth factor-induced ischemic hindlimb collateralization and nitric oxide-mediated angiogenesis. Circulation Research 2004;95:154-161.

79. Morais C, Ebrahem Q, Anand-Apte B, Parat MO. Altered angiogenesis in caveolin-1 gene-deficient mice is restored by ablation of endothelial nitric oxide synthase. The American Journal of Pathology 2012;180:1702-1714.

80. Sbaa E, Dewever J, Martinive P, Bouzin C, Frerart F, Balligand $\mathrm{JL}$, et al. Caveolin plays a central role in endothelial progenitor cell mobilization and homing in sdf-1-driven postischemic vasculogenesis. Circulation Research 2006;98:1219-1227.

81. Navarro G, Borroto-Escuela DO, Fuxe K, Franco R. Potential of caveolae in the therapy of cardiovascular and neurological diseases. Frontiers in Physiology 2014;5:370.

82. Jasmin JF, Malhotra S, Singh Dhallu M, Mercier I, Rosenbaum DM, Lisanti MP. Caveolin-1 deficiency increases cerebral ischemic injury. Circulation Research 2007;100:721-729.

83. Xu L, Guo R, Xie Y, Ma M, Ye R, Liu X. Caveolae: Molecular insights and therapeutic targets for stroke. Expert Opin Ther Targets 2015; 19:633-650.

84. Fujimura M, Shimizu H, Inoue T, Mugikura S, Saito A, Tominaga T. Significance of focal cerebral hyperperfusion as a cause of transient neurologic deterioration after extracranial-intracranial bypass for moyamoya disease: Comparative study with non-moyamoya patients using n-isopropyl-p-[(123)i]iodoamphetamine single-photon emission computed tomography. Neurosurgery 2011;68:957-964; discussion 964-965.

85. Bang OY, Goyal M, Liebeskind DS. Collateral circulation in ischemic stroke: Assessment tools and therapeutic strategies. Stroke 2015;46:3302-3309. 